\title{
Análisis sistémico de la comunidad y el individuo en el proceso de reintegración paramilitar en Colombia (2003-2006)*
}

\author{
Recibido: 03 de octubre del 2018• Aprobado: 11 de noviembre del 2018 \\ https://doi.org/10.22395/ojum.v17n35a10 \\ Diana Alejandra Mateus García"* \\ Milena Alexandra González Piñeros ${ }^{* * *}$
}

\begin{abstract}
RESUMEN
Los procesos de reintegración competen tanto a las personas que se encuentran en ellos como a la sociedad que está pasando por tal coyuntura. Tras el análisis de la Ruta de Reintegración aplicada en el lapso 2003-2006 para la desmovilización paramilitar en Colombia, surge el interrogante acerca de sus implicaciones en el momento en el que alguien se enfrenta a un cambio de realidad y la forma en la cual la sociedad es partícipe en este proceso. Mediante un estudio cualitativo, se analizan cuatro historias de vida tomadas del programa de radio "La Reintegración, el camino hacia la paz", las cuales se contrastan con las dimensiones para la reintegración: personal, habitabilidad y ciudadanía, diseñados desde el enfoque individual en el papel de los excombatientes y el enfoque comunal en la disposición de la sociedad. Como resultado, se argumenta que es necesario incorporar una articulación sistémica para el éxito del proceso.
\end{abstract}

Palabras clave: Colombia; excombatiente; enfoque comunal; enfoque individual; paramilitarismo; reintegración.

Este artículo es el resultado del trabajo de investigación desarrollado en el espacio académico de Investigación Asistida de la Universidad Santo Tomás. Surge con la intención de hacer una revisión conceptual y la necesidad coyuntural en el país de profundizar la investigación sobre contextos de postconflicto.

** Estudiante de pregrado en Gobierno y Relaciones Internacionales de la Universidad Santo Tomás. Joven gestora en y para la investigación de la Universidad Santo Tomás, auxiliar de investigación en proyecto sobre desplazamiento forzoso. Áreas de investigación: seguridad urbana y grupos post- desmovilización, reintegración comunitaria y procesos de memoria colectiva. Correo electrónico: diana.mateus@usantotomas.edu.co Orcid: https://orcid.org/0000-0001-6511-6838

.*. Abogada de la Universidad Nacional de Colombia, especialista en Derechos Humanos de la Universidad Andina Simón Bolívar (Ecuador), magíster en Economía Social de la Universidad Nacional de General Sarmiento (Argentina), magíster en Investigación Social Interdisciplinaria de la Universidad Distrital Francisco José de Caldas (Colombia) y candidata a Doctora en Ciencia Política de la Escuela de Política y Gobierno de la UNSAM (Argentina). Docente del área de Investigación de la Facultad de Gobierno y Relaciones Internacionales, Universidad Santo Tomás (Colombia). Investigadora del Grupo de Estudios en Gobierno y Relaciones Internacionales (Gegri). Áreas de investigación: ciencia política, metodologías, derecho constitucional, economía social, sociología jurídica. Correo electrónico: milenagonzalez@usantotomas.edu. co Orcid: https://orcid.org/0000-0002-0929-9569 


\title{
Systemic Analysis of the Community and the Individual during the Paramilitaries' Reintegration Process in Colombia (2003-2006)
}

\begin{abstract}
Reintegration processes are as much a matter for the people who are going through them as for the society that is living such a conjuncture. After the analysis of the Ruta de Integración (Reintegration Route) applied between 2003 and 2006 for the paramilitary demobilization in Colombia, a arises about its implications at the moment in which someone faces a change of reality and about the way in which the society participates in this process. With a qualitative study, four life stories taken from the radio program "La Reintegración, el camino hacia la paz" (Reintegration, the path to peace) are analyzed and contrasted with the dimensions for reintegration: personal, habitability and citizenship. The mentioned dimensions are designed from an individual perspective on the role of ex-combatants and a communal approach regarding society's disposition. As a result, we argue that it's necessary to incorporate a systemic articulation for the process to be successful.
\end{abstract}

Keywords: Colombia; ex-combatant; community approach; individual approach; paramilitarism; reintegration.

\section{Análise sistêmica da comunidade e do indivíduo no processo de reintegração paramilitar na Colômbia (2003-2006)}

\section{RESUMO}

Os processos de reintegração competem tanto às pessoas que participam deles quanto à sociedade que está passando por tal conjuntura. Após a análise da Rota de Reintegração aplicada no intervalo de 2003-2006 para a desmobilização paramilitar na Colômbia, surge a questão sobre suas implicações no momento em que alguém encara uma mudança de realidade e a forma na qual a sociedade é partícipe nesse processo. Por meio de um estudo qualitativo, quatro histórias de vida do programa de rádio "La Reintegración, el camino hacia la paz" (A Reintegração, o caminho para a paz) são analisadas. Tais histórias são contrastadas com as dimensões para a reintegração: pessoal, habitabilidade e cidadania, desenhadas a partir da abordagem individual no papel dos ex-combatentes e na abordagem municipal na disposição da sociedade. Como resultado, argumenta-se que é necessário incorporar uma articulação sistêmica para o sucesso do processo.

Palavras-chave: Colômbia; ex-combatente; abordagem municipal; abordagem individual; paramilitarismo; reintegração. 


\section{INTRODUCCIÓN}

La política de reintegración aplicada en Colombia es en estos días un tema de gran relevancia, debido a la coyuntura por la que está atravesando el país al presentarse una de las desmovilizaciones más grandes con el grupo Fuerzas Armadas Revolucionarias de Colombia (De ahora en adelante FARC-EP). A partir de este suceso, surge una de las preguntas que más resuenan en la sociedad: ¿cómo se va a recibir a los excombatientes? Se hace necesario adoptar una mirada retrospectiva y analítica sobre la manera en la cual se han llevado a cabo las políticas de reintegración y los aspectos positivos que se pueden obtener de estas experiencias, no solo como un ejemplo de los beneficios que obtienen los excombatientes al abandonar las armas, sino también de las retribuciones para la sociedad al implementar estas estrategias.

La Agencia Colombiana para la Reintegración (ACR) establece específicamente

\footnotetext{
"La ACR es una entidad adscrita a la Presidencia de la República, que está encargada de coordinar, asesorar y ejecutar [...] la Ruta de Reintegración de las personas desmovilizadas de los grupos armados al margen de la ley" (ACR, 2016a). Actualmente, esta institución tiene como nombre Agencia Colombiana para la Normalización y la Reincorporación y se modificó mediante el Decreto Ley 897 del 29 de mayo de 2017. A lo largo de este trabajo, se utilizará como referencia la ACR debido a que fue específicamente bajo esta institución que se llevaron a cabo las medidas para la reintegración masiva de los excombatientes de las Autodefensas Unidas de Colombia y las condiciones para la reintegración son distintas a las que se contemplan ahora en el proceso de reincorporación y normalización.

Adicionalmente, la ACR diseña, implementa y evalúa la política de Estado dirigida a la Reintegración social y económica de las personas o grupos armados al margen de la ley que se desmovilicen voluntariamente, de manera individual o colectiva" (ACR, 2016a).
}

ocho dimensiones de la Ruta de Reintegración (ACR, 2016), mediante las cuales se hacen explícitos los criterios necesarios para hacer exitoso este proceso. Sin embargo, en este artículo se hizo un contraste de tres de esas dimensiones, ya que son, por consideración en categoría de análisis, las más idóneas para mostrar el vínculo existente entre el enfoque individual y el comunal para la superación personal de las personas en proceso de desmovilización.

De esta manera, se seleccionaron las dimensiones de reintegración: personal, habitabilidad y ciudadanía, las cuales están correlacionadas entre sí al estar contempladas en el enfoque individual y comunal, lo que lleva a determinar que el éxito de este proceso está implícito, tanto en la disposición de la persona que inicia el proyecto, como en la sociedad en la cual se materializan las experiencias de interacción colectiva y por tanto, la disposición de toda una comunidad para que se haga efectiva una reinserción efectiva.

Se empleó un método cualitativo apoyado en recolección de documentos que explican el proceso de reintegración y las dinámicas bajo las cuales se requiere como base a la sociedad para hacer efectivo su funcionamiento. También se emplearon cuatro narrativas de experiencias de reintegración emitidas en el programa de radio "La Reintegración, el camino hacia la paz"2, un espacio de la

\footnotetext{
"El programa de radio de la Agencia Colombiana para la Reintegración (ACR) pretende hacer pedagogía frente a la construcción de paz, la reconciliación y la reintegración de personas desmovilizadas. Para ello, en el programa se
} 
Agencia Colombiana para la Reintegración en el que se brinda la oportunidad a los excombatientes de contar su experiencia en el proceso de reintegración y cómo llevó a cabo la Ruta de Reintegración para finalmente graduarse y ser reconocido como un ciudadano reintegrado a la sociedad colombiana.

Tras la descripción cualitativa de las dimensiones y las historias de vida de cuatro excombatientes narradas en el programa de radio "La Reintegración, el camino hacia la paz", se determinó que es necesario incorporar una articulación sistémica como rubro para la relación, tanto de las dimensiones como de los enfoques pues este es un factor que ayuda a consolidar de manera más práctica el enfoque comunal y el enfoque personal; uno de los principales problemas que encontraron las personas pertenecientes al programa de reintegración fue la aceptación de su condición anterior en la sociedad y por ello, la importancia de preparar el entorno para que comprenda los cambios que se darán en él y así lograr exitosamente la culminación del proceso.

Por tanto, el aporte que deja el estudio de las tres dimensiones trabajadas es la implementación de un modelo sistémico para llevar a cabo la Ruta de la Reinte-

traslada la responsabilidad de estas temáticas a toda la sociedad en general [...] Por lo anterior, los invitados de 'La Reintegración, el camino hacia la paz' son ciudadanos de diferentes sectores: políticos, actores, músicos, artistas, deportistas, representantes de la fuerza pública, de la iglesia, de las ONG, funcionarios públicos, personas desmovilizadas, víctimas del conflicto, periodistas, y académicos [...]". (ACR, 2016b). gración ${ }^{3}$, ya que con este tipo de rúbrica se incluirá a la sociedad en el proceso y hará posible dejar a un lado los sesgos existentes sobre la reincorporación a la vida civil de los excombatientes. Ellos a su vez, tendrán una noción positiva de la percepción general que tienen las personas sobre ellos, lo cual genera mucha más confianza en el programa de reintegración.

\section{METODOLOGÍA}

La técnica usada para la recolección de datos es la recopilación de cuatro historias de vida ${ }^{4}$, en las cuales los protagonistas relatan cómo fue su proceso de reintegración desde el momento en que iniciaron hasta el día de su graduación, ya que a partir de las voces de estas personas se puede hacer una caracterización de cada una de las dimensiones que

"La Ruta de Reintegración se define como el conjunto de condiciones, beneficios, estrategias, metodologías y acciones definidos por la Agencia Colombiana para la Reintegración de Personas y Grupos Alzados en Armas, concertados con la persona en proceso de reintegración, para promover el desarrollo de capacidades, la superación de la situación de vulnerabilidad y el ejercicio autónomo de la ciudadanía. [...

Al diseñarse la ruta de un participante en el Proceso de Reintegración se busca que, mediante un ejercicio de concertación entre esa persona y la ACR, se obtenga un esquema de actividades acorde con las opciones reales del individuo en relación con el proyecto de vida deseado, sin perder de vista la forma como están reglamentados los beneficios sociales, económicos y jurídicos del Proceso de Reintegración" (ACR, 2016c).

4 Las historias de vida relatadas en el programa de radio "la Reintegración, el camino hacia la paz" son las narrativas de las personas que pertenecieron al programa de reintegración y que culminaron con éxito su proceso, durante el cual las personas contaron con todo tipo de herramientas y objetivos para que pudiesen culminar este camino y llegar al día de su graduación, un acto simbólico de gran valor para quienes hicieron parte de este programa (ACR, 2016b). 
componen la Ruta de la Reintegración. Por ello, este es un estudio de tipo cualitativo y descriptivo; a partir de las voces de quienes hicieron parte del procedimiento, fue posible describir las dimensiones y la influencia que tienen en cada uno de los enfoques.

Para el desarrollo de la presente investigación, en primera instancia se hizo un análisis de documentos expuestos por la Agencia Colombiana para la Reintegración (ACR) con el fin de determinar cuáles son las dimensiones que se abordan en los procesos de reintegración de los excombatientes; de acuerdo con esto, se categorizaron las dimensiones de la siguiente manera:

1. Personal, en la cual se enmarca la importancia de la salud física y mental de la persona que inicia el proceso de reintegración y que resulta crucial trabajar para la construcción de una identidad individual que permita una re-significación de los relatos con los cuales las personas comprenden su experiencia de vida. El estudio de esta dimensión se consideró importante porque permite hacer un análisis psicosocial del individuo y así determinar su posición frente a la realidad contextual.

2. Habitabilidad, la cual comprende la inclusión en un sentido familiar y las formas en las cuales se relaciona con ese círculo de personas en su ambiente más cercano a partir del desarrollo de capacidades que mejoren sus condiciones de vida.
3. Ciudadanía, la cual da una idea de estatus para la participación y el cumplimiento de los derechos tal que se comience a dar la legalidad en relación con la sociedad y el reconocimiento del Estado y las instituciones.

Luego de la comprensión alcanzada sobre las tres dimensiones, se hace un contraste con las narrativas de cuatro personas que fueron entrevistadas en el programa de la Agencia Colombiana para la Reintegración (ACR). En sus relatos explicaban cómo había sido su proceso de reintegración con respecto a cada una de las dimensiones que trabajaron en su proceso, los desafíos a los cuales se habían tenido que enfrentar, las dificultades que tuvieron, las capacidades que desarrollaron, las competencias que auto- descubrieron en cada etapa, su posición actual frente a la sociedad y al proceso de reintegración que se llevó a cabo con ellos y su perspectiva sobre los mecanismos aplicados con otras personas que se adscribieron al proceso.

Siendo así, se prestó especial atención a los momentos en los cuales las personas narraron cómo inició su proceso individual, la persona que eran antes de ingresar al programa y quienes son ahora debido a la resignificación de su identidad y las diferentes destrezas que encontraron en ellos mismos, tanto para superar esa etapa de violencia que tuvieron que vivir como para sentirse personas útiles para la sociedad.

Para caracterizar la dimensión desde el punto de vista de las personas que hicie- 
ron parte del proceso de reintegración, también se tuvo en cuenta su interacción con la comunidad y con los diferentes grupos sociales que los acogieron para llevar a cabo su proceso. En este punto, los criterios de selección estuvieron guiados por conceptos como comunidad, cooperación y oportunidades, ya que es de esta manera como se puede entender a la sociedad desde el punto de vista de quien hace parte de un proceso de reintegración.

Por otro lado, la dimensión de ciudadanía a abordó a partir de elementos como institución, trabajo y asistencia, pues con estos es posible determinar la posición de la persona sobre ellos en el proceso de reintegración, si los concibe como conceptos diferenciados o si por el contrario le resultan congruentes o necesarios de forma interconectada para poder culminar de manera exitosa su proceso de reintegración.

Finalmente, con base en los criterios mencionados por las personas que narraron su historia de vida en el proceso de reintegración en Colombia, se estructura un cuadro de tipo cíclico y sistémico que explica la relación existente entre las dimensiones y enfoques con la realidad que tiene que vivir la persona que lleva a cabo el proceso para aportar así una mirada más cohesionada de las partes que lo conforman. A continuación se presenta la tabla usada para la recolección de la información.

Para facilitar la compresión de las variables teóricas, seguidamente se incluye un gráfico que orienta la comprensión sistémica de la Ruta de la Reintegración, el cual se elaboró con base en la información institucional de la ACR y se reinterpretó para efectos de la investigación.

Tabla 1. Sistematización para la recolección de información

\begin{tabular}{ll}
\hline & Habitabilidad Ciudadanía Personal \\
\hline Enfoque \\
comunal
\end{tabular}

Fuente: elaboración propia con base en la información de la ACR

\section{CONDICIONES PREVIAS AL PARAMILITARISMO EN COLOMBIA}

Para comprender el tema central de este artículo, es necesario hacer un recorrido histórico por las estructuras, situaciones y coyunturas que condicionaron el nacimiento del paramilitarismo y las Autodefensas Unidas de Colombia (AUC). Uno de estos primeros momentos, comprende La Violencia, un proceso en el que las grandes esferas políticas crearon un acuerdo llamado Frente Nacional que consistía en la alternación del poder administrativo entre los dos grandes partidos tradicionales (Liberal y Conservador) con el fin de mantener una estabilidad política. Sin embargo, la poca respuesta y eficiencia del Estado ante diferentes problemáticas sociales y su falta de presencia en zonas periféricas del país, llevó a que se generaran grupos definidos como autodefensas 


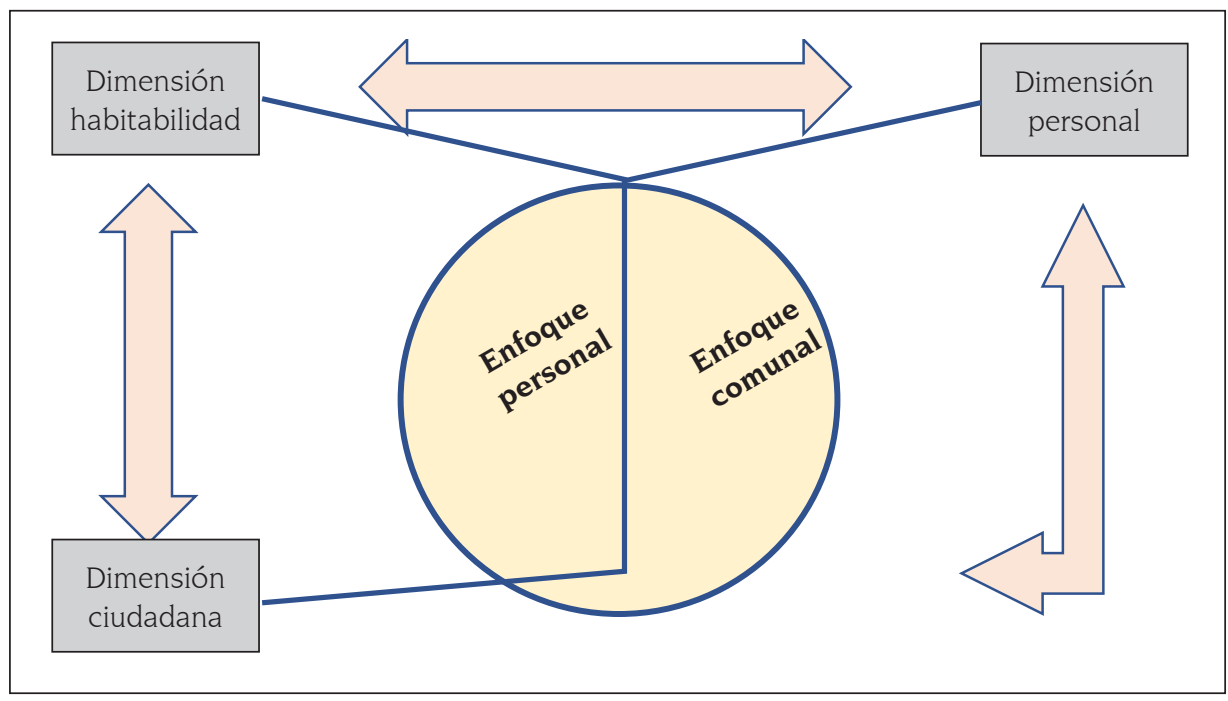

Figura 1. Comprensión sistémica de la Ruta de la Reintegración

Fuente: elaboración propia con base en la información de la ACR

campesinas. Estas tenían como finalidad una revolución que favoreciera a la clase campesina, pero posteriormente adoptaron bases ideológicas que apoyaban la causa de suplantar al Estado. Finalmente se consolidaron guerrillas como: Ejército colombiano de Liberación Nacional (ELN), Ejército Popular de Liberación (EPL), Movimiento 19 de abril (M-19) y Fuerzas Armadas Revolucionarias de Colombia Ejército del Pueblo (FARC-EP).

Debido a la expansión y gran impacto que generaron estos grupos insurgentes, durante los años ochenta surgieron los grupos paramilitares como una fuerza contrainsurgente con una ideología anticomunista, apoyados por las élites políticas que buscaban asegurar sus intereses. Aunque en un principio los intereses eran privados, posteriormente el gobierno colombiano creó el Estatuto de Seguridad y De- fensa de la Democracia en 1978 que contenía dentro en su estructura, los criterios para combatir a los grupos guerrilleros en pro de una Defensa Nacional para la seguridad civil y por tanto, la creación de autodefensas, las cuales poco a poco se fueron consolidando con doctrina y entrenamiento para luego ser armados por el mismo Estado colombiano para que la ciudadanía sirviera como apoyo para combatir contra la guerrilla (Velásquez, 2007).

Posteriormente, con el aumento de las dinámicas guerrilleras en la década de 1990, en el gobierno de César Gaviria Trujillo se emite el Decreto Ley 3567 de 1994 en el cual se dan los parámetros para regular los "grupos especiales de seguridad privada" Convivir, es decir, cooperativas de vigilancia y seguridad privada.

En este punto, los grupos paramilitares 
ya conformaban una seguridad privada respaldada por el gobierno que además contaba con el apoyo de grandes terratenientes. Posteriormente se unieron a los grupos Convivir para crear las AUC en 1996 como un grupo de extrema derecha distribuido en 16 grupos a lo largo del país cuyo jefe era Carlos Castaño. Igualmente, contaban con el respaldo de ganaderos, terratenientes y con el surgimiento del narcotráfico, de un financiamiento adicional que les permitió proliferarse de manera más eficiente, tanto en cuanto a cantidad de personas pertenecientes a sus filas como en cuanto a capacidad armamentística, la cual adquirieron gracias al aumento de sus ingresos.

Los nexos con el narcotráfico no solo enriquecieron a las AUC al momento de la expansión de su dominio territorial, posteriormente, la tenencia de estos cultivos les permitió vender lo que ellos mismos llamarían "franquicias" a narcotraficantes reconocidos y así disminuir estos territorios y poder negociar con el gobierno de Estados Unidos las condiciones de su extradición. (Puentes, 2008).

Con el pasar del tiempo, esta organización comenzó a ser mucho más visible y sus métodos para acabar con los movimientos guerrilleros estaban marcados por una fuerte línea de crueldad. En muchos de los casos, estas acciones no fueron ocasionadas en contra de las fuerzas guerrilleras sino de la población civil a la que, de manera arbitraria consideraban simpatizantes de algún grupo insurgente. Tras la llegada de Álvaro Uribe Vélez a la Presidencia en el 2002, las AUC iniciaron una serie de conversaciones y se comenzaron a plantear estudios para generar diálogos y llegar a un acuerdo para el cese de la actividad paramilitar, para lo que el gobierno nombró a la Comisión Exploratoria de Paz el 23 de diciembre del 2002. Unos meses después se suscribió el Acuerdo de Santa Fe de Ralito, con el cual se inició el proceso de negociación (Centro Nacional de Memoria Histórica, 2012).

En el Acuerdo de Santa Fe de Ralito se pactó la desmovilización de la totalidad de los militantes. Este proceso empezó en el 2003, para lo cual el Congreso de la República decidió aprobar la Ley de Justicia y Paz que contiene los parámetros para hacer más fáciles los procesos de paz y la reincorporación a la vida civil de los excombatientes -en ese momento, de aquellos pertenecientes a las AUC- y buscar que se garantice el derecho a la verdad, justicia y reparación de las víctimas. Además de esto, también se creó la Comisión Nacional de Reparación y Reconciliación (CNRR) y la Agencia Colombiana para la Reintegración (ACR), esta última es una institución que tiene como fin preparar programas para la desmovilización, el desarme y la reintegración de los excombatientes a la vida civil y así prevenir que reincidan en procesos de violencia o que muten a grupos de crimen organizado. 


\section{CONDICIONES ACTUALES DE ESTUDIO}

Como se mencionó anteriormente, en la actualidad se está llevando a cabo un proceso de desmovilización que conduce al interrogante sobre si el realizado anteriormente con las AUC aplica en este momento de la historia colombiana, ya que se espera que en el caso presente las cifras sean mucho más favorables y que a partir de la experiencia anterior, las instituciones tengan los soportes y las bases suficientes para fundamentar y llevar a cabo el proceso de manera más eficiente.

En la actualidad resulta necesario desarrollar estudios de profundidad que ayuden a comprender los factores que hacen parte del proceso de reintegración y en específico, de los enfoques individual y comunal, con el fin de comprender la dirección y los lineamientos de las dimensiones que trabaja la Ruta para la Reintegración. Esto puesto que se hace evidente una preocupación generalizada en la población sobre cómo se van a llevar a cabo las reincorporaciones a la sociedad de los actuales excombatientes. A pesar de que se conocen los parámetros públicamente, aún la sociedad no tiene un conocimiento amplio sobre el proceso, lo que ha generado reticencia en la sociedad hacia estas personas, que en muchas ocasiones también pueden ser catalogadas como víctimas de diferentes situaciones de violencia estructural que, de una u otra manera, condicionaron su actuar.
Por tanto, es importante que todas las instituciones formales que hacer parte de este proceso se adapten a las nuevas necesidades, tanto de las personas reintegradas como de la sociedad en general; tal que esta última comprenda cuál es su papel en este momento crucial para el país y no caiga en viejos errores de estigmatización y discriminación hacia esta población por falta de información o poco desarrollo de las políticas que ayuden a la comprensión de la situación especial de los individuos.

En ese sentido, también es importante mencionar que la ACR ha tenido un avance significativo en la inclusión de estrategias que mejoren la institucionalidad en los territorios, debido a que la presencia insuficiente de organismos estatales ha sido un factor de análisis para comprender la presencia de grupos irregulares que, de manera poco eficiente, intentan suplantar al Estado (Hernández-Delgado, 2013).

Las dimensiones bajo las cuales la Agencia Colombiana para la Reintegración inicia a una persona en este proceso son ocho: habitabilidad, ciudadanía, productiva, personal, seguridad, salud, educación y familiar; todas hacen parte de un procedimiento en el cual a la persona se le dan las herramientas suficientes y los apoyos necesarios para que se reincorpore en la sociedad como alguien productivo y que contribuya, tanto a su bienestar personal como al fortalecimiento de lazos que hayan sido dañados por tantos años de conflicto. 
En este artículo se plantea un análisis sistémico de las dimensiones correspondientes tanto al enfoque comunal como al individual, ya que con la comprensión de esta perspectiva sobre el proceso, se hace posible cohesionar el entorno con cada una de las dimensiones a trabajar y por tanto, se incluye también a la sociedad en la preparación y posterior adaptación al desarrollo de los programas necesarios para la reintegración.

\section{FUNDAMENTOS TEÓRICOS PARA LA DIMENSIÓN DE HABITABILIDAD EN LA REINTEGRACIÓN}

La ACR comprende el concepto de habitabilidad, como un concepto que va más allá de la simple mirada que se le da un hábitat determinado para un individuo, ya que, comprende que también está relacionado con las diferentes dinámicas que se dan en un grupo social, esto, debido a que los seres humanos no solo necesitan una estructura física para convivir, sino que también son necesarias las relaciones que se dan en determinado ambiente.

Parte además, de la premisa sobre las condiciones óptimas que debe tener un hábitat para que se desarrollen correctas relaciones humanas. Se necesita un acondicionamiento, tanto de la estructura física, como de las estructuras sociales, ya que es por medio de la interacción con otras personas que se logra la comprensión de los diferentes fenómenos y es de esta forma que la persona que inicia un proceso de reintegración, puede comenzar a entender cuáles son los nuevos modelos a los cuales se debe adaptar para ir cumpliendo los objetivos que le plantea la Ruta de Reintegración. (ACR, 2016C).

Al adoptar una visión amplia sobre lo que implica la dimensión de habitabilidad en el proceso de reintegración, un análisis teórico dese el enfoque histórico-sociológico se hace posible. Este permite comprender las relaciones de los sujetos en determinado entorno de forma diacrónica, lo cual facilita reconocer las tendencias que caracterizan esas relaciones humanas. Luego a partir de la comprensión de estas relaciones, se puede hacer una comparación que determine cuál es la forma más idónea de hacer viable la relación entre los individuos pertenecientes a un entorno determinado (Losada y Casas, 2008).

Para la comprensión del enfoque histórico sociológico vale la pena analizar diferentes autores que, por medio de sus investigaciones, facilitan una caracterización del enfoque. En ese sentido, según Charles Tilly (1977) en From mobilization to revolution, existen cinco grandes elementos para la acción colectiva: las organizaciones, los intereses, las oportunidades, la acción colectiva y la movilización. Cada uno de estos componentes resultan esenciales para entender el comportamiento y las tendencias de los individuos en un entorno determinado. Aunque el autor hace estas caracterizaciones a grandes rasgos para comprender de qué manera es que la sociedad, de manera colectiva, se une para un objetivo determinado, también es posible identificar allí la importancia que 
le da al acondicionamiento del ambiente y a la predisposición de los sujetos para acondicionar el medio.

Desde el punto de vista del concepto de habitabilidad, la acción colectiva se puede definir como las dinámicas con las cuales las personas que pertenecen al proceso de reintegración comienzan a hacer parte de un nuevo entorno; en este también deben interactuar con nuevas organizaciones, hacer parte de unos intereses diferentes a los que tenían en su vida como combatientes y a su vez, tener nuevas oportunidades para así poder modificar su comportamiento y adaptarse de manera más sencilla al entorno.

Por otro lado, en Institutions politiques et droit constitutionnel, Duverger (1970) hace un recorrido por las instituciones políticas de Francia para luego hacer una comparación de su funcionamiento con respecto a las máximas consagradas en el derecho constitucional y como por medio de la instauración de las diferentes normativas, ha sido posible direccionar ciertos comportamientos en la sociedad. Es decir que por medio de la creación de normas, se ha llevado a cabo también la extensión de diferentes instituciones políticas que permitieron, en un primer momento, suscitar comportamientos formales que enseñaron a la sociedad a tener determinadas conductas. Apuntala Duverger también que con el tiempo, la misma tradición y articulación de las conductas sociales con el entorno lograron establecer instituciones no formales que de una manera u otra, permitieron que las personas comprendieran el entorno, ya no desde un sentido normativo, sino que de manera consuetudinaria pudieron adaptarse al entorno como también crear incentivos para que el resto de la sociedad también lo hiciera.

En las narraciones de los excombatientes pertenecientes al programa de reintegración de la ACR tomadas del programa de radio "La Reintegración, el camino hacia la paz", es posible notar que, por ejemplo, ellos siguen un conducto, unos lineamientos normativos que, aunque no están establecidos en la Constitución Política, son bajo los cuales deben regir su comportamiento; sin ellos no sería posible llevar a cabo el proceso de reintegración. Sin embargo, el programa no cuenta con la extensión a la sociedad para que se cumpla con la predisposición del ambiente tal que las personas que ahora hacen parte del proceso de reintegración puedan relacionarse inmediatamente con las reglas de juego, ellos mismos deben buscar la forma de comenzar a pertenecer a las instituciones informales.

En este artículo se adopta la perspectiva del enfoque de Charles Tilly (1977) debido a que hace un análisis diacrónico y sincrónico al mismo tiempo sobre cómo funcionan los componentes que permiten la acción colectiva y la influencia que tienen los individuos y el ambiente sobre los cambios que en él se efectúen. Esto se puede interpretar en la dimensión de habitabilidad, lo que lleva a comprender la habitabilidad no solo como la forma en la cual debe vivir la persona que inicia el 
proceso de reintegración -lo cual incluye una vivienda digna, la convivencia con su familia y con la comunidad - sino también las condiciones del hábitat para que la persona conozca las nuevas perspectivas que van a hacer parte de su vida de ahora en adelante en su círculo más cercano y que también la sociedad comprenda cómo debe incluir al individuo en estas, pues como lo explica el enfoque, aprender de las viejas tendencias lleva a que se encuentren mejores alternativas para el desarrollo de un proceso.

\section{FUNDAMENTOS TEÓRICOS PARA LA DIMENSIÓN DE CIUDADANÍA EN LA REINTEGRACIÓN}

Esta dimensión se entiende como la capacidad que desarrolla la persona que hace parte del proceso de reintegración de ser reconocido con un estatus en el cual se abordan el goce de los derechos y el cumplimiento de deberes, tal y como lo hace cualquier ciudadano. Esta dimensión implica la relación de las personas pertenecientes al programa con el resto de la sociedad en el marco de la legalidad. Al haber pertenecido a un grupo que imponía su propio "marco legal", dejaron por fuera de su diario vivir el reconocimiento de las instituciones y de todas aquellas funciones fundamentales a las cuales todos los sujetos pertenecientes a un orden constitucional están sometidos (ACR, 2016d).

De acuerdo con esta caracterización de la dimensión de ciudadanía, se puede inferir que el enfoque jurídico-institucional es el más idóneo para analizar este momento en el proceso de reintegración. Esto debido a que dentro de los términos que más utiliza para sus estudios está la legitimidad y la autoridad del Estado, de sus políticas y sus instituciones, así como también las diferentes maneras en que los individuos siguen la norma de acuerdo con la legitimidad que esta tiene para generar algún cambio en el comportamiento de la población, y la interacción con las instituciones jurídicas y políticas que conforman el orden nacional.

El enfoque se encarga de estudiar la importancia de la institución y la manera en la cual se relaciona con las demás instituciones, como también la importancia de su existencia para corresponder de manera legítima o normativa a la sociedad. Lo anterior también supone la recepción (positiva o de rechazo) de la población de dichas reglas de juego. En la dimensión de ciudadanía, esto se traduce en la generación de instituciones que tienen como fin ayudar a las personas en su proceso de reintegración a la vida civil, pero que también trae consigo una serie de parámetros que la persona debe comenzar a hacer parte de su realidad, como el reconocimiento de la autoridad del Estado y las instituciones pertenecientes a él, e incluso en una perspectiva más individual, en el estatus de participación que se ofrece al individuo (Losada y Casas, 2008).

Además, está la participación en los procesos de justicia transicional en el aspecto normativo, para que comiencen a tener una visión acerca de la legalidad jurídica 
y una valoración de los compromisos que adquieren al desmovilizarse, que van mucho más allá de la esfera personal pues se convierten en grandes esferas de responsabilidad social. Con esto, es posible comprender que la dimensión de ciudadanía no solo le otorga a la persona un reconocimiento por parte del Estado, sino que también le atribuye ciertas características para que le sea posible desarrollar su proceso de reintegración con éxito. Sin embargo, la dificultad para acceder a estas instituciones jurídicas sigue siendo un tema ensombrecido por la misma falta de respuesta de muchas de ellas que van perdiendo poco a poco la confianza de las personas vinculadas al programa.

Uno de los autores que ha trabajado el enfoque jurídico-institucional es Douglass C. North (1991), quien explica que las instituciones son, en definitiva, una invención que tiene como fin establecer los parámetros centrales para controlar la acción humana y así también, establecer determinados parámetros por medio de la cultura y las costumbres. No obstante, el individuo también necesita una retribución dentro de esas normas de juego (formales e informales) para que, por un lado, la institución consiga un consenso en la sociedad y por otro lado, el orden jurídico corresponda a las necesidades y a los códigos de conducta de la población. Es así como la sociedad influye en el cumplimiento o no de determinadas acciones.

Al relacionar la dimensión de ciudadanía en la Ruta de la Reintegración, la nor- mativa y los procesos establecidos para conseguir la reintegración exitosa de los excombatientes, es necesario establecer ciertos criterios para dar una mirada nueva a estas personas. Por ello, se crean las situaciones jurídicas óptimas como por ejemplo, la justicia transicional para que los excombatientes colaboren con las víctimas en el esclarecimiento de lo sucedido y así tener la verdad sobre los hechos, o los programas comunales para que comiencen a reconocerse dentro de la sociedad como ciudadanos y a las demás personas como parte de un mismo orden.

Otro autor que ha trabajado este enfoque es Herman Finer, en su libro The Theory and Practice of Modern of Government expone que las normas y el orden jurisdiccional por sí solo no pueden establecer los parámetros necesarios e idóneos para que la sociedad se configure en torno a ellos; se necesita la implementación de herramientas prácticas que ayuden a la consolidación de los parámetros para que así exista una completa armonía entre lo que la norma quiere establecer y lo que la sociedad entiende de ella. Estas herramientas se entenderían como las instituciones que pueden incentivar a los individuos a hacer parte de esta nueva forma de comprensión de los mecanismos de organización (Munro, 1932).

Si se aplica lo mencionado anteriormente a la dimensión de ciudadanía, se puede inferir que en el proceso de reintegración es necesario implementar herramientas que ayuden a fundamentar las medidas institucionales y jurídicas que comprende esta dimensión. De no ser así, no sería 
posible que las personas en proceso de reintegración pudieran terminarlo de manera exitosa, ni que comprendieran del todo lo que implica seguir este tipo de normativas.

En este artículo se sigue la perspectiva de Douglass C. North (1991) la cual tiene en cuenta los papeles principales de las instituciones y de la organización jurídica, así como la importancia de la existencia de herramientas para su consolidación. También presenta la idea de que estos mecanismos deben existir de manera acorde con la necesidad de cada sociedad y de cada contexto; es necesario que tanto las organizaciones como la sociedad puedan comprender estos códigos y que de esta manera sea mucho más fácil que compaginen adecuadamente con los individuos. Lo anterior puede llevar a comprender el hecho de que tanto la normativa y las herramientas que se utilicen en la reintegración deben estar encaminadas a la comprensión del contexto y que la sociedad debe comprender alguna forma de retribución y así encontrar un incentivo para corresponder a estos códigos.

\section{FUNDAMENTOS TEÓRICOS PARA LA DIMENSIÓN PERSONAL EN LA REINTEGRACIÓN}

En tanto a la dimensión personal, se hace un análisis a partir del enfoque psicosocial pues este se plantea estudiar las diferentes dinámicas que se dan en el mundo a partir del interior de las personas. Es decir que se centra en encontrar cómo el individuo percibe los fenómenos sociales y cómo por medio de su propia experiencia puede explicarlos y relacionarse con ellos. En este sentido, los factores psicológicos también van a condicionar la postura de la persona en la sociedad y su forma de comprender las situaciones en el contexto en que se encuentra inmerso. Por lo anterior, las personas que hacen parte del proceso de reinserción deben ser comprendidas a partir de estas premisas, pues la forma en la cual ellos se han relacionado con el mundo es diferente al de las personas que no han estado inmersas en el conflicto, e incluso al de una víctima del mismo (Losada y Casas, 2008).

Cuando las personas acceden a hacer parte del proceso de desmovilización y posteriormente comienzan a llevar a cabo la Ruta para la Reintegración, traen consigo diferentes aspectos psicológicos que posteriormente van a condicionar su forma de actuar. Los programas utilizados para trabajar la esfera personal están encaminados a la búsqueda de una identidad individual a partir de la identificación colectiva para que la persona pueda sentir empatía con determinado grupo social. Para ello, es necesario resignificar los relatos de estas personas, no como una justificación sino como una comprensión de las situaciones que le condicionaron.

De los autores que plantean este enfoque es posible nombrar a Pippa Norris y Ronald Inglehart (2004), editores de Sacred and secular: Religion and politics worldwide, en el cual examinan cada una de las incidencias del contexto cultural y socio-económico que determinan los niveles bajo a altos 
de percepción de seguridad ciudadana y de creencia política, todo esto lo hacen mediante un estudio cuantitativo con encuestas realizadas a más de ochenta países en el mundo; logran operativizar los conceptos personales que utilizan los individuos para definir su percepción del entorno en el cual se encuentran, así como la significación de sus modos de comportamiento a partir de la identidad individual.

Bajo esta perspectiva, se puede comprender la dimensión personal como las nociones que tienen las personas en el proceso de reintegración con respecto a la sociedad. Es a partir de esta visión que propondrán la visión de cómo percibir la forma en la cual la sociedad los ve y asimismo, cómo se consideran de alguna manera necesarios en ese entorno al que pertenecen. Esto debido a que, en el modelo de reintegración, no solo se trabaja lo correspondiente a las situaciones traumáticas por las que hayan pasado, sino también cómo crear condiciones óptimas para que estos individuos hagan parte de la sociedad.

Por otro lado, también se encuentra el estudio The Oxford Handbook of Political Behavior (2007), un estudio realizado por Russell J. Dalton y Hans Dieter Klingemann en el cual estudian las variables incidentes en la participación política y los progresos con respecto a la creencia de los ciudadanos en los modelos sociales y la comunicación que llega a la sociedad. También estudia cómo los modos estructurales forman la opinión pública, pero al mismo tiempo, el individuo es partícipe en esa estructura para la consolidación de un modelo macro de pensamiento.

De acuerdo con lo anterior, se puede comprender la dimensión personal a partir de los procesos mediante los cuales ayudan a las personas que hacen parte de la Ruta de Reintegración basados en la concepción que estas tengan sobre ese nuevo contexto en el que se encuentran. Dicha concepción difiere sustancialmente de las creencias que tenían sobre las instituciones o la sociedad en general cuando hacían parte del grupo paramilitar si se compara con como ahora enfrentan las nuevas realidades de un contexto fuera de las filas.

Este artículo adhiere a la perspectiva de Pippa Norris y Ronald Inglehart (2004) en la cual se incluyen también los aspectos psicológicos de las personas frente a sus condiciones anteriores a la coyuntura, adecuados a su entorno socio-económico. De tal manera, se posibilita determinar los lazos necesarios para intentar dar una explicación sistémica del proceso comunal y personal pues, hay muy poca información sobre su desarrollo y, a pesar de que no se encuentra completamente especificada su realización, las personas en sus narrativas parecen comprender mejor el trabajo que hacen a nivel individual. Ellos mismos comienzan a hacer una relación directa entre la persona que eran antes de ingresar al programa y quienes son después de culminar su Ruta para la Reintegración y ser reconocidos en su graduación como personas completamente nuevas. 


\section{LA RELACIÓN DEL PENSAMIENTO SISTÉMICO CON LAS DIMENSIONES DE LA RUTA PARA LA REINTEGRACIÓN}

Por último, este artículo plantea que para comprender, tanto en el enfoque individual como en el comunal, los criterios que corresponden a cada una de las dimensiones, es necesario abordarlas desde una perspectiva sistémica, que sugiere la apropiación de los entornos, los ambientes y los contextos para com prender y correlacionar las dinámicas que se desarrollan en estos. De esta manera se entiende el por qué los problemas al momento de la reintegración, como lo es específicamente la posición de la sociedad que no conoce sobre la ruta que deben llevar a cabo las personas que decidieron desmovilizarse y por ende tampoco comprende las dimensiones en una estructura de sistema sino que por el contrario, los lee como factores diferentes entre ellos. Asimismo, a pesar de que la ruta que expone la Agencia Colombiana para la Reintegración intenta hacer una cohesión entre ellas, tampoco las gestiona como un solo cuerpo sino como una lista de chequeo con la que se "evalúa" la capacidad de respuesta de la persona frente al momento del proceso en el que se encuentra.

Siendo así, para el análisis sistémico de la reintegración en el caso de Colombia, es necesario tener en cuenta la perspectiva de David Easton (1965) en Categorías para el análisis sistémico de la política en el cual, hace una aproximación a cuáles son los procesos por los que debe pasar un sis- tema para que logre persistir en el tiempo y siga respondiendo de forma efectiva a las necesidades de los individuos pertenecientes al entorno.

Easton también establece que los sistemas son mecanismos abiertos en los cuales se garantiza la retroalimentación de las diferentes propuestas puestas en práctica y los resultados positivos o negativos que estas tienen en su implementación, ya que así es posible evaluar su efectividad.

En la aplicación de la Ruta de la Reintegración, específicamente en las dimensiones de habitabilidad, ciudadanía y personal, es posible establecer qué es necesario para el correcto funcionamiento de estas variables en el enfoque personal y con mucho más trabajo, en el comunal. Esto debido a que el éxito de estas dimensiones está dada por la respuesta de aceptación con la cual la sociedad incluya a la persona en proceso de reintegración. Es así que también es necesaria la adaptabilidad del entorno para que las personas que próximamente entren en el proceso, puedan tener una percepción más alta de la capacidad de adaptación tanto de ellos con el ambiente como del ambiente para con ellos.

Por tanto, el estudio de las relaciones entre las variables que hacen parte de un proceso, como lo explica David Easton (1965), ayuda a que se gestionen nuevas soluciones a los problemas, ya que, con una estructura sistémica, existe un factor de retroalimentación que permite que se 
corrijan los errores que se pudieron dar en la ejecución de alguna de las demandas. Así bien, la retroalimentación de las personas que hicieron parte del proceso de reintegración es un factor esencial para corregir los baches entre las dimensiones que ayudan a correlacionar el enfoque individual y el comunal para garantizar el éxito del proceso de reintegración.

De acuerdo con el planteamiento central de este artículo, el análisis sistémico de la reintegración es posible desde los relatos en los que se evidencian las dimensiones de habitabilidad, ciudadanía y personal, con respecto al enfoque comunal y personal. En las narraciones de las personas que desarrollaron con éxito su ruta de reintegración y que decidieron compartir su historia en el programa de radio "La Reintegración, el camino hacia la paz" se pueden identificar estas relaciones desde sus propias palabras: "[...] no es sencillo dejar las armas e iniciar un nuevo camino pero hay que hacerlo a pesar de que la sociedad muchas veces lo rechace a uno por su condición" (historia de vida José Edilberto García, 17 de marzo 2017)

Como resultado de la sistematización de la información recogida de las narraciones de los excombatientes que pertenecieron a grupos paramilitares, se encuentra que:

a. Al hablar del concepto de habitabilidad, a pesar de que se entienda como la capacidad de vivir y comprender nuevos espacios, a las personas que pertenecieron al programa de reintegración les presentó la dificultad de comprender el entorno. Esto debido a la poca aceptación que recibieron por parte de la población. Como la sociedad no conoce o no se interesa en conocer sobre el proceso que las personas deben pasar, sienten rechazo hacia ellas y esto dificulta que se puedan dar interacciones normales en el hábitat y que las personas reintegradas verdaderamente comiencen a sentirse parte de nuevo de la sociedad.

A lo largo del programa del 28 de marzo de 2017, el señor Edwin Cárdenas Ochoa luego de su desmovilización, encontró en la panadería su hobby, y el modo de sostener a su familia. Edwin compartió su historia de vida en el programa "La Reintegración, el camino hacia la paz" y es posible encontrar en esta el proceso por el cual tuvo que pasar para que la sociedad lo recibiera de una forma más cordial y como un ciudadano más y no como un reintegrado. Cárdenas explica que al principio fue difícil mencionar el hecho de que estaba en proceso de reintegración pues existe el miedo al rechazo, las palabras más destacadas en su testimonio frente a la comunidad son rechazo, estigmatización y miedo.

Posteriormente, relacionarse fue uno de los pasos que lo ayudó para continuar en su proceso, por lo cual, ahora él se identifica socialmente de la siguiente manera: "Tengo mucha energía y después de aprender el negocio de la panadería me di cuenta que soy muy alegre y que tengo mucho que aportarle a la comunidad" (historia de vida Edwin Cárdenas Ochoa, 28 de marzo 2017, emisión Santa Marta). 
Se alude entonces a la perspectiva del enfoque de Charles Tilly (1977) debido a su análisis diacrónico y sincrónico sobre cómo funcionan los componentes que permiten la acción colectiva y la influencia que tienen los individuos y el ambiente sobre los cambios que en él se efectúen. Esto se puede ver en la dimensión de habitabilidad y lleva a comprender la habitabilidad no solo como la forma en la cual debe vivir la persona que inicia el proceso de reintegración -vivienda digna, convivencia con su familia y con la comunidad- sino también las condiciones del hábitat, tal que la persona conozca las nuevas perspectivas que van a hacer parte de su vida de ahora en adelante en su círculo más cercano y también la sociedad comprenda cómo debe incluir al individuo en estas. Como lo explica el enfoque, aprender de las viejas tendencias lleva a que se encuentren mejores alternativas para el desarrollo de un proceso.

b. El concepto de ciudadanía ha cumplido con su objetivo de formar a las personas reintegradas en la legalidad y el reconocimiento a las instituciones estatales. Sin embargo, uno de los principales factores para el reconocimiento como ciudadano es también la percepción de los otros, lo cual resulta ser un impedimento, tanto para las personas que se reintegraron como para la sociedad; no se ha dado un afianzamiento entre quienes hicieron parte del programa de reintegración y la sociedad en general porque siguen existiendo factores de desconfianza y de poca información de las dos partes para consolidar verdaderamente un sentido de ciudadanía.

En la emisión del 17 de marzo de 2017 el programa expuso cómo la reintegración le dio la oportunidad a José Edilberto García de tener su tienda. Él es uno de los 939 reintegrados del Magdalena que han recibido capital semilla por parte de la ACR para fortalecer o montar su negocio, y que, a partir de esto, se ha dado cuenta de que el programa de reintegración otorga oportunidades óptimas para conseguir un futuro mejor.

Es así como establece que

La ACR me ha ayudado mucho, todos los doctores, los especialistas, se centran en que uno de verdad se sienta bien y pueda salir adelante, a mí me dieron la oportunidad de mejorar mi vida crediticia y pude sacar un crédito para poner mi tienda, ahora las personas no me ven como reintegrado sino como el señor de la tienda. (historia de vida José Edilberto García, 17 de marzo 2017)

Con estas herramientas la dimensión de ciudadanía consolida la importancia del reconocimiento del Estado y las instituciones que hacen parte de él. Como también mencionaba Juan Guillermo en el programa del 8 de abril de 2016: "La entidad fue muy cumplida porque nos cumplieron a nosotros con todo lo que prometieron, pero la gente muchas veces no entiende el proceso por el que uno pasa" (historia de vida Juan Guillermo, 8 de abril de 2016). 
Se retoma la perspectiva de Douglass C. North (1991) en "Institutions, Journal of Economic Perspectives", la cual tiene en cuenta los papeles principales de las instituciones y de la organización jurídica, así como también la importancia de la existencia de herramientas para su consolidación. Que adicionalmente esgrime que estos mecanismos deben existir de acuerdo a la necesidad de cada sociedad y contexto, pues es necesario que tanto las organizaciones como la sociedad puedan comprender estos códigos y sea de esta manera mucho más fácil que se relacionen adecuadamente con los individuos. De acuerdo con esto se puede comprender que la normativa y las herramientas que se utilicen en la reintegración deben estar encaminadas a la comprensión del contexto y que la sociedad debe percibir alguna forma de retribución y así encontrar un incentivo para corresponder a estos códigos.

c. El concepto de dimensión personal es entendido como la manera en la cual ellos logran superar los problemas psicológicos con los que llegan después de tantos años en la guerra. También se le entiende como la capacidad de interactuar con las demás personas, de relacionarse como individuos comunes en los grupos y de mostrar a las personas las diferentes capacidades que poseen, y que pueden ser útiles para los demás.

El programa del 7 de octubre de 2016 cuenta la historia de Neco, un desmovilizado de las AUC que culminó con éxito su proceso de reintegración y hoy es un miembro activo de su comunidad. Neco afirma que: "Ya uno aprende de la comprensión, la tolerancia, porque yo antes de ser así como estoy, era muy rebelde y no entendía muchas cosas, pero ya con el programa la psicóloga me apoyó mucho". (ACR, 2016b).

Se retoma el análisis de Pippa Norris y Ronald Inglehart (2004) que incluye también los aspectos psicológicos de las personas con respecto a sus condiciones anteriores a la coyuntura y adecuados con su entorno socio-económico que permitan establecer los lazos necesarios para una explicación sistémica del proceso comunal y personal. Esto debido a que hay muy poca información sobre su desarrollo y a pesar de que no se encuentra completamente especificada su realización, las personas en sus narrativas parecen comprender mejor el trabajo que se hace a nivel individual. Ellos comienzan a hacer una relación directa entre la persona que eran antes de ingresar al programa y quienes son después de culminar su Ruta para la Reintegración y ser reconocidos en su graduación como personas completamente nuevas.

Las personas que pertenecieron al programa de reintegración conocían la ruta de desarrollo para el éxito de su proceso, sin embargo, la forma en la cual llevaron a cabo su proceso no busca solucionar dimensión por dimensión, sino que supone una estructura conjunta que ellos comienzan a implementar para hacer efectivo su proceso. 
d. La caracterización sistémica de las dimensiones de ciudadanía, habitabilidad y personal con respecto a los enfoques: comunal y personal, permite una comprensión más amplia de los criterios que comprenden las personas que culminaron el proceso de reintegración. Por tanto, ofrece una mirada más amplia para lograr una cohesión entre la sociedad y el programa, porque se definen los puntos específicos en los cuales se cruzan las visiones sociales con las perspectivas de las personas reintegradas. Esto no solo permite mejorar las condiciones del proceso de reintegración sino que también dota de herramientas sólidas a las comunidades para entender mejor este proceso.

\section{CONCLUSIONES}

A partir de las definiciones dadas por la Agencia Colombiana para la Reintegración (ACR) en las dimensiones entendidas como: ciudadanía, habitabilidad y personal, es importante resaltar que cada uno de estos conceptos y lo que conlleva es comprendido por las personas que hicieron parte del programa. No obstante, en la práctica y el desarrollo de su proceso se comienzan a entender otros factores y criterios que influyen en cómo se lleva a cabo la ruta de reintegración la cual no necesariamente está organizada para desarrollar las dimensiones de manera aislada.

En primera medida está la dimensión personal -que se definió como la importancia de la salud física y mental de la persona que inicia el proceso de reintegración - en la que resulta muy importante trabajar en la construcción de una identidad individual para resignificar los relatos con los cuales las personas comprenden su experiencia de vida. Allí se encuentran dos factores importantes, por un lado, la construcción de identidad que -como se expuso en este artículo- supone un proceso de interacción. Esto debido a que la identidad nace de una serie de procesos de relación entre el individuo con la sociedad y sus prácticas, para que a partir de esto se identifique dentro de un grupo social y por tanto culmine su proceso de creación de identidad. Esto se resume en el hecho de que las personas pertenecientes al proceso de reintegración necesitan una esfera social en la que puedan construir su identidad sin ser rechazados por su condición.

Por otro lado, se enmarca también la resignificación de los relatos con los cuales las personas comprenden su experiencia de vida. Este es un componente importante para dar a la persona bases para fortalecer su autoestima y la visión que tiene sobre sí mismo. No obstante, en las narraciones se encontró una tendencia a explicar este factor como la búsqueda de un rol para esta persona en la sociedad. Más allá de comprender su experiencia de vida de una forma menos traumática para el individuo, se trata del papel que desempeñan ahora en la sociedad y del valor que se les otorga, ya no como reintegrados sino como personas útiles; no se concibe necesario comprender de otra forma su vida, sino de dar un sentido 
nuevo y que la sociedad les permita demostrar el interés que tienen por ser personas normales y que no se les tilde por su condición anterior.

En un segundo punto se encuentra el concepto de habitabilidad comprendido como la inclusión en un sentido familiar y las formas en las cuales se relaciona ese círculo en su ambiente más cercano a partir del desarrollo de capacidades que mejoren sus condiciones de vida. Sin embargo, a partir del enfoque bajo el cual se estudió este concepto, las condiciones del entorno están dadas también por la interacción que los sujetos puedan construir con las unidades pertenecientes a ese entorno o hábitat. Es debido a esto que las personas explicaban en sus narraciones que una de las partes más difíciles en el proceso era la aceptación de la sociedad, en muchas ocasiones tenían que ocultar su verdad para no ser rechazados en el lugar en el que vivían o trabajaban. Es por esto que la condición de habitabilidad no solo debe estar en la disposición de adaptarse de la persona que hace parte del proceso de reintegración, sino también en el acondicionamiento y preparación de la sociedad.

En el tercer concepto de análisis, el de ciudadanía, se comprendió la idea de estatus para la participación y el cum plimiento de los derechos para que se comience a dar una relación de legalidad con la sociedad y el reconocimiento del Estado y las instituciones. Esta definición, en contraste con el enfoque jurídicoinstitucional, responde a las unidades de análisis para ser una dimensión importante de desarrollar en el sentido de la construcción de un modelo de legalidad y legitimidad en la persona reintegrada. No obstante, la comprensión de ciudadanía del individuo está condicionada por algo más que el contrato social, esta se determina también por el reconocimiento que le otorguen los demás ciudadanos como una parte perteneciente al mismo grupo y no como "el otro"; lo que pone en duda la aplicación de esta dimensión en la población en general, ya que no responde al sentido de integración que propone el programa de reintegración.

Estas nuevas formas de comprender el proceso de reintegración han dotado a las comunidades de la información necesaria para aceptar e incluir en los territorios a las personas que inician el proceso de reintegración, lo cual indica que ha existido una transformación en la forma en la cual se comprende al excombatiente. Como se expone a continuación, las tensiones entre las comunidades se pueden solucionar por medio de la relación natural y por tanto sistémica que existe en los entornos sociales.

Finalmente, las categorías descriptivas que surgen en este análisis de las tres dimensiones, tales como aceptación, perdón, paz, entendimiento, cambio, integración, cooperación, oportunidades, ayuda, asistencia, cambio; y también las de rechazo, miedo, vergüenza, estigmatización y desconfianza son el resultado de: a) la interacción entre las dimensiones, aunque no sea de manera 


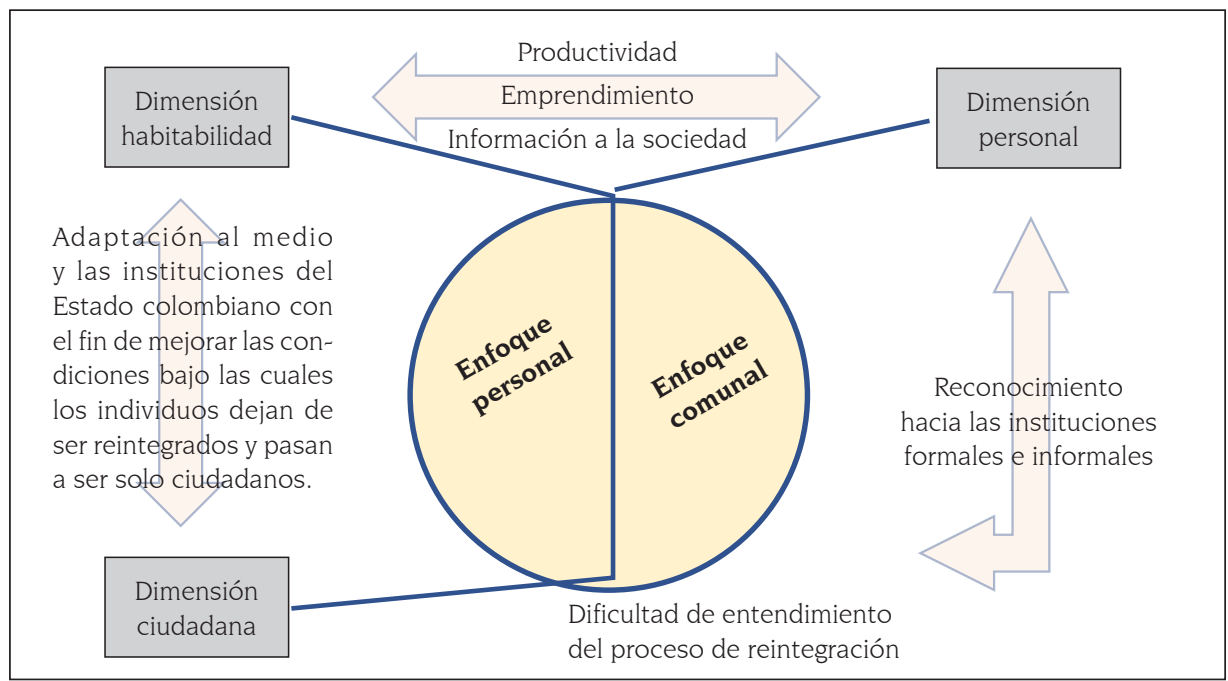

Figura 2. Sistematización de los resultados: comprensión sistémica de la Ruta para la Reintegración Fuente: elaboración propia con base en la información de la ACR

voluntaria, pero que por la naturaleza sistémica del proceso, funcionan de forma articulada y no aislada una de la otra, pues en las dinámicas de interacción poco a poco se van dando esas relaciones; y b) las categorías que describen la falta de preparación e inclusión de la sociedad en los procesos de reintegración. La distancia que existe entre el entorno y las personas es considerablemente alta y por ello se generan las indisposiciones y luego, el rechazo a esta población, lo que indudablemente lleva a que se ponga en riesgo el proceso de la persona y la eficiencia en la forma de entender el programa para la reintegración en Colombia.

\section{REFERENCIAS}

Abuljanova Slavskaia, K. (1988). La correlación entre lo individual y lo social. En Problemas teóricos de la psicología de la personalidad (pp. 147-152). La Habana: Orbe.
Agencia Colombiana para la Reintegración (ACR). (2016). Dimensiones. Bogotá, Colombia: Agencia para la Reincorporación y la Normalización. Recuperado de http://www. reintegracion.gov.co/es/la-reintegracion/ Paginas/dimensiones.aspx

Agencia Colombiana para la Reintegración (ACR). (2016a). Acerca de la ACR. Bogotá, Colombia: Agencia para la Reincorporación y la Normalización. Recuperado de http://www. reincorporacion.gov.co/es/agencia/Paginas/ acerca-de.aspx

Agencia Colombiana para la Reintegración (ACR). (2016b). Programa de Radio. Bogotá, Colombia: Agencia para la Reincorporación y la Normalización. Recuperado de http://www. reincorporacion.gov.co/es/sala-de-prensa/ Paginas/radio.aspx?Paged=TRUEEp _ Ar ticleStartDate $=20161112+05: 00: 00 \varepsilon p$ SortBehavior $=0 \varepsilon p$ ArticleStartDate $=20$ 161112+05:00:00Ep Title=La+invitaci\% C3\%B3n+de+Juan+Manuel+Gal\%C3\%Al $\mathrm{n}+\mathrm{a}+\mathrm{la}+$ reconciliaci\%C3\%B3n\&p $\quad \mathrm{ID}=11$ 82EPageFirstRow $=16 \& E$ View $=\% 7$ BB0487 2AC-64E3-4552-AFC5-BAE73E730267\%7D 
Agencia Colombiana para la Reintegración (ACR). (2016c). Ruta de Reintegración. Bogotá, Colombia. Agencia para la Normalización y la Reincorporación. Recuperado de http://www. reincorporacion.gov.co/es/la-reintegracion/ Paginas/ruta.aspx

Agencia Colombiana para la Reintegración (ACR). (2016d). Dimensión ciudadana. Bogotá, Colombia: Agencia para la Reincorporación y la Normalización. Recuperado de http://www. reintegracion.gov.co/es/la-reintegracion/ Paginas/ciudadana.aspx

Casas, R. L. (2008). Enfoques para el análisis político. Bogotá, Colombia: Pontificia Universidad Javeriana.

Centro Nacional de Memoria Histórica (2012). Justicia y Paz: Tierras y territorios en las versiones de los paramilitares. Informe del Centro Nacional de Memoria Histórica. Bogotá, Colombia: $\mathrm{CNMH}$.

D’Angelo Hernández, O. (2003). Enfoque histórico-cultural, complejidad y desarrollo humano en una perspectiva integradora, transdisciplinaria y emancipatoria Imemorias en cd]. Encuentro Internacional Hóminis-02. La Habana, Cuba: Centro de Investigaciones Psicológicas y Sociológicas (CIPS).

Dalton, R., y Klingemann, H-D. (2007). The Oxford Handbook of political Behavior. Oxford: Oxford University Press.

Duverger, M. (1961). Méthodes des Sciences sociales. París: Presses Universitaires de France.

Duverger, M. (1970). Institutions politiques et droit constitutionnel. París: Presses Universitaires de France.

Easton, D. (1965). Categorías para el análisis sistémico de la política. Nueva York: Knopf, Inc.

Hernández Delgado, E. (2013). Mediaciones en el conflicto armado colombiano. Hallazgos desde la investigación para la paz. CONfines de relaciones internacionales y ciencia política,
9(18), 31-57. Recuperado de http://www.scielo.org.mx/scielo.php?script=sci_abstract Epid $=$ S1870-35692013000200002Elng $=$ es Enrm=iso

Hodgson, G. M. (2011, julio-diciembre). ¿Qué son las instituciones? CS, (8), 17-53. https://doi. org/10.18046/recs.i8.1128

Munro, W. (1932, agosto). The Theory and Practice of Modern Government by Herman Finer [reseña]. The American Political Science Review, 26(4), 728-730. doi: https://doi. org/10.2307/1946543

Norris, P., e Inglehart, R. (2004). Sacred and secular: Religion and politics worldwide. Cambridge: Cambridge University Press.

North, D. (1991). Institutions. Journal of Economic Perspectives, 5(1), 97-112. Recuperado de https://edisciplinas.usp.br/pluginfile. $\mathrm{php/1695541/ \textrm {mod } \text { resource/content/1/ }}$ North\%20\%281991\%29.pdf

Puentes, A. M. (2008, octubre). Narcotráfico y su papel en el desmonte de los paramilitares. Verdad Abierta. Recuperado de https://verdadabierta.com/narcotrafico-y-su-papel-en-eldesmonte-de-los-paramilitares/

Tilly, C. (1977). From mobilization to revolution. Michigan: University of Michigan.

Velásquez Rivera, E. J. (2007). Historia del paramilitarismo en Colombia. História (São Paulo), 26(1), 134-153. Recuperado de http://www.scielo.br/scielo.php?pid=S0101$90742007000100012 \mathrm{Escript}=\mathrm{sci}$ abstractEtlng=es

Verdad abierta. (2009) Carlos Castaño Gil [perfill. Recuperado de https://verdadabierta.com/ perfil-de-carlos-castano-gil/ 